\title{
Model Based Diagnosis of Both Sensor-Faults and Leakage in the Air-Intake System of an SI-Engine
}

\author{
Mattias Nyberg \\ Vehicular Systems, ISY, Linköping University \\ S-581 83 Linköping, Sweden \\ e-mail: matny@isy.liu.se \\ phone: +4613282369 \\ fax: +4613282035
}

\begin{abstract}
Many model based solutions to diagnosis problems in SIengines have been discussed in literature. However most presented methods are useful only for a specific class of faults. Here a systematic and more general method is presented. With this method, which is based on a structure of hypothesis tests, it is possible to diagnose a large variety of different types of faults. The method is applied to the diagnosis of sensor-faults and leakage in the air-intake system of an SI-engine. The features of the method are demonstrated by using experiments on a real SI-engine. The experiments show that the method is capable to diagnose both leakage and different types of sensor faults. Both detection and isolation are considered. It is for example possible to distinguish between a manifold leak and a manifold pressure sensor fault.
\end{abstract}

\section{INTRODUCTION}

On-board diagnosis of car engines has become increasingly important because of environmentally based legislative regulations such as OBDII (On-Board Diagnostics) [1]. Other reasons for incorporating diagnosis in vehicles are repairability, availability and vehicle protection. Today, up to $50 \%$ of the engine management systems are dedicated to diagnosis.

One important area of SI (Spark Ignition) engine diagnosis is the diagnosis of all sensors connected to the airintake system. Another important problem is the diagnosis of leakage in the air intake system. These two diagnosis problems were investigated separately in two previous papers [2] and [3] respectively. In both papers, the principle of model based diagnosis was used and although the technique is not fully developed, it is obvious from these results and also other works, that there is much to gain by using a model based approach to diagnosis of SI-engines.

In the first paper [2], sensor faults were modeled as additive signals and the diagnosis method used the principle of structured residuals [4] in combination with diagnostic observers. In the second paper [3], leakages were physically modeled as air-flows through restrictions and the diagnosis problem became a parameter estimation problem. The two papers show that each solution works well and good performance is obtained. However, the two solutions are so different in nature that they can not easily be combined into a single diagnosis system that can diagnose both types of faults simultaneously.

Thus the challenge, and the objective of this paper, is to find a systematic diagnosis method that is able to solve both the sensor and the leakage diagnosis problem. One of the issues that needs to be addressed is the isolation problem, i.e. to distinguish between different faults. For instance, a leakage can easily be mis-interpreted as a airmass flow sensor fault if not extra care is taken.

The diagnosis method presented here is general and includes the previous solutions as special cases. The basic framework is a structure of hypothesis tests and the general ideas are described in Section 2. The goal then, is to demonstrate these ideas on the air-intake system of a real production engine. The engine is described and modeled in Sections 3 and 4. This particular engine is turbo-charged but all results are also valid for a naturally aspirated SIengine. In Section 5, the general diagnosis ideas are further developed and a working diagnosis system is presented. Section 6 contains a validation on the real engine and it is shown that the diagnosis system is able to distinguish between different faults, e.g. leakage and sensor faults. Finally Section 7 deals with on-line implementation issues and Section 8 contains the conclusion.

\section{MODEL BASED DIAGNOSIS USING STRUC- TURED HYPOTHESIS TESTS}

This section presents a new general method for model based diagnosis. The approach is to use a structure of hypothesis tests. As a result of these general ideas, the model of the system can be fully utilized in a systematic way. This implies that it is possible to diagnose a large variety of different types of faults. 


\subsection{Fault Modes}

For constructing a model based diagnosis system, a model of the process is needed. This model must contain the process behavior in the fault free case and also include a definition of different faults and how faults affect the process. We classify the different faults into fault modes. For example all manifold leaks, regardless of their area, belong to the fault mode "manifold leak" which we will denote $M L$. Another fault mode is the case "no fault" which we will denote $N F$.

The exact fault of the process is described by the fault state $\theta$, which mostly is vector valued. The set of all possible fault states is denoted $\Theta$ and the different fault modes divides $\Theta$ into disjunct subsets $\Theta_{\gamma}$. Thus, each fault mode $\gamma$ is associated with a subset $\Theta_{\gamma}$. In addition, each fault mode $\gamma$ is associated with a model of the process $\mathcal{M}_{\gamma}\left(\theta_{\gamma}\right)$, where $\theta_{\gamma}$ is a part of the vector $\theta$. For each fault mode, the other parts of the vector $\theta$, are assumed to be fixed to some constant. That is, if we know that the fault mode of the process is $\gamma$ and also that $\theta_{\gamma}$ has a fixed value, then the fault state $\theta$ is uniquely specified. The parameter space of $\theta_{\gamma}$ for the case fault mode $\gamma$ is present, will be denoted $\Xi_{\gamma}$. The set of all models, i.e. $\mathcal{M}(\theta)=\left\{\mathcal{M}_{\gamma}\left(\theta_{\gamma}\right)\right\}$ is the total model needed to construct the diagnosis system.

For an example, consider a system described by the following equations:

$$
\begin{aligned}
\dot{x} & =f(x, u) \\
y_{1} & =h_{1}(x)+b_{1} \\
y_{2} & =h_{2}(x)+b_{2} \\
b_{1} & \geq 0 \\
b_{2} & \geq 0
\end{aligned}
$$

The constants $b_{1}$ and $b_{2}$ represents sensor bias faults and it is assumed that only positive biases can occur. Three fault modes are considered: no fault $N F$, a bias in sensor $1 B 1$, and a bias in sensor $2 B 2$. The fault state of the system is described by the vector $\theta=\left[\begin{array}{ll}b_{1} & b_{2}\end{array}\right]$. The model associated with fault mode $B 1$ is $\mathcal{M}_{B 1}\left(b_{1}\right)$ and obtained from (1) by fixing $b_{2}=0$. That is, we have that $\theta_{B 1} \equiv b_{1}$. The parameter space $\Xi_{B 1}$ becomes $\left.] 0, \infty\right]$. Similarly the model associated with fault mode $B 2$, obtained from (1) by fixing $b_{1}=0$, is $\mathcal{M}_{B 2}\left(b_{2}\right)$ and the parameter space $\Xi_{B 2}$ becomes ] $0, \infty]$. The model associated with fault mode $N F$ is $\mathcal{M}_{N F}$ and obtained from (1) by fixing $b_{1}=b_{2}=0$. Thus in this case, there is no parameter $\theta_{N F}$.

\subsection{Structured Hypothesis Tests}

This section describes a principle of structured hypothesis tests which can be seen as a generalization of the well known principle of structured residuals [4]. Using the principle of structured hypothesis tests, the diagnosis system has a structure according to Figure 1. The inputs to the diagnosis system is the process input $u$ and the process

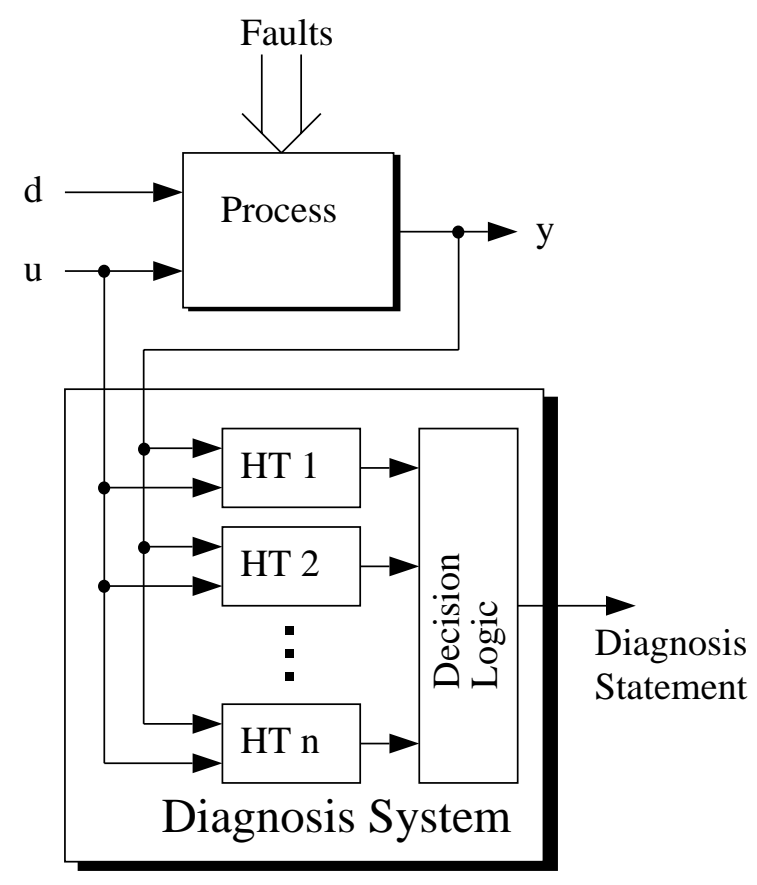

Figure 1 The diagnosis system using the principle of structured hypothesis tests.

output $y$. The signal $d$ represents inputs that are unknown to the diagnosis system, e.g. disturbances. The output of the diagnosis system is the diagnosis statement, which contains information of which fault modes that can explain the behavior of the process. Then the diagnosis system consists of a set of hypothesis tests, HT 1 to $H T$ $n$, and the decision logic.

The null hypothesis for the $i$ :th hypothesis test, i.e. $H_{i}^{0}$, is that the fault mode, present in the process, belongs to a specific set $M_{i}$ of fault modes. The alternative hypothesis $H_{i}^{1}$ is that the present fault mode does not belong to $M_{i}$. This means that if hypothesis $H_{i}^{0}$ is rejected, and thus $H_{i}^{1}$ is accepted, the present fault mode can not belong to $M_{i}$. In this way, each separate hypothesis test contributes with a piece of informations about which fault modes that can be present. The purpose of the decision logic is then to combine this information to form the diagnosis statement.

For example, let $F_{p}$ denote the present fault mode and assume that the diagnosis system contains the following set of three hypothesis tests:

$$
\begin{array}{ll}
H_{1}^{0}: F_{p} \in M_{1}=\left\{N F, F_{1}\right\} & H_{1}^{1}: F_{p} \in M_{1}^{C}=\left\{F_{2}, F_{3}\right\} \\
H_{2}^{0}: F_{p} \in M_{2}=\left\{N F, F_{2}\right\} & H_{2}^{1}: F_{p} \in M_{2}^{C}=\left\{F_{1}, F_{3}\right\} \\
H_{3}^{0}: F_{p} \in M_{3}=\left\{N F, F_{3}\right\} & H_{3}^{1}: F_{p} \in M_{3}^{C}=\left\{F_{1}, F_{2}\right\}
\end{array}
$$

Then if only $H_{1}^{0}$ is rejected, we can draw the conclusion that $F_{p} \in M_{1}^{C}=\left\{F_{2}, F_{3}\right\}$, i.e. the present fault mode is either $F_{2}$ or $F_{3}$. If both $H_{1}^{0}$ and $H_{2}^{0}$ are rejected, we can draw the conclusion that $F_{p} \in M_{1}^{C} \cap M_{2}^{C}=\left\{F_{2}, F_{3}\right\} \cap$ $\left\{F_{1}, F_{3}\right\}=\left\{F_{3}\right\}$, i.e. the present fault mode is $F_{3}$. 
From the above example, it is clear that the decision logic is a simple intersection operation. The diagnosis statement $S$ then becomes a set of fault modes that can be expressed as

$$
S=\bigcap_{\substack{i \\ H_{i}^{1} \text { accepted }}} M_{i}^{C}
$$

From this definition of $S$, it is obvious that the diagnosis statement can contain more than one fault mode. This feature corresponds well to a desired functionality since in cases where it is difficult or even impossible to decide which fault mode has occured, it is very useful for a service technician to get to know that there are more than one fault mode that can explain the behavior of the process. If the diagnosis system was forced to pick out one fault mode in cases like this, it is highly probable that a mistake is made and wrong fault mode is picked out.

Much of the engineering work involved in constructing a diagnosis system is to use the model $\mathcal{M}(\theta)$ to construct the individual hypothesis tests. How the hypothesis tests are constructed depends on the actual case and only for some specific classes of systems, general design procedures have been proposed, e.g. linear systems. The actual design of hypothesis tests for the air-intake system will be covered in Section 5.1.

\section{EXPERIMENTAL SETUP}

All experiments were performed on a 4 cylinder, 2.3 liter, turbo-charged, spark-ignited SAAB production engine. It is constructed for the SAAB 9-5 model. The engine is mounted in a test bench together with a Schenck "DYNAS NT 85" AC dynamometer. Both during the model building and the validation, the engine were run according to Phase I+II of the FTP-75 test-cycle. The data for the test cycle had first been collected on a car with automatic transmission.

Leaks were applied by using exchangeable bolts. One bolt were mounted in the wall of the manifold and the other in the wall of the air tube in front of the throttle. The exchangeable bolts had drilled holes of different diameters ranging from $1 \mathrm{~mm}$ to $8 \mathrm{~mm}$.

Data were collected by a DAQ-card mounted in a standard PC. All data were filtered with a LP-filter with a cutoff frequency of $2 \mathrm{~Hz}$.

\section{AIR-INTAKE SYSTEM MODEL}

A schematic picture of the air-intake system is shown in Figure 2. Ambient air enters the system and an air-mass flow sensor measures the air-mass flow rate $\dot{m}$. Next, the air passes the compressor side of the turbo-charger and then the intercooler. This results in a boost pressure $p_{b}$ and temperature $T$ that is higher than ambient pressure and temperature respectively. Next, the air passes the throttle and the flow $\dot{m}_{t h}$ is dependant on $p_{b}, T$, the throttle angle $\alpha$, and the manifold pressure $p_{m}$. Finally the air leaves the manifold and enters the cylinder. This flow $\dot{m}_{c y l}$ is dependant on $p_{m}$ and the engine speed $n$. Also shown in the figure are the two possible leaks: the boost leak somewhere between the air-mass flow sensor and the throttle, and the manifold leak somewhere in the manifold.

In this work, the air-intake system is modeled by a mean value model [5]. This means that no within cycle variations are covered by the model. Because there is no need for extremely fast detection of leakage, it is for the model, sufficient to consider only static relations. The modeling work, including identification of parameters, was presented in [3]. Below we give a short description of the model for the fault-free system and also the models of the leakages.

\subsection{Fault-Free Model}

The model for the fault-free system is described by the following equations

$$
\begin{aligned}
& \dot{m}=\dot{m}_{t h} \\
& \dot{m}_{t h}=\dot{m}_{c y l} \\
& \dot{m}_{t h}=f\left(p_{b}, \alpha, p_{m}\right)=\frac{C_{d} A_{t h} p_{b}}{\sqrt{R T}} \Psi\left(\frac{p_{m}}{p_{b}}\right) \\
& A_{t h}=A_{1}\left(1-\cos \left(a_{0} \alpha+a_{1}\right)\right)+A_{0} \\
& \Psi\left(\frac{p_{m}}{p_{b}}\right)=\left\{\begin{array}{c}
\sqrt{\frac{2 \kappa}{\kappa-1}\left\{\left(\frac{p_{m}}{p_{b}}\right)^{\frac{2}{\kappa}}-\left(\frac{p_{m}}{p_{b}}\right)^{\frac{\kappa+1}{\kappa}}\right\}} \\
\text { if }\left(\frac{p_{m}}{p_{b}}\right) \geq\left(\frac{2}{\kappa+1}\right)^{\frac{\kappa}{\kappa-1}} \\
\sqrt{\kappa\left(\frac{2}{\kappa+1}\right)^{\frac{\kappa+1}{\kappa-1}}} \quad \text { otherwise }
\end{array}\right. \\
& \dot{m}_{c y l}=g\left(p_{m}, n\right)
\end{aligned}
$$

The equations describing the throttle air-flow $\dot{m}_{t h}$ are the commonly used equations describing flow through a restriction [6] [7]. From now on, it is assumed that the temperature $T$ is constant, which explains why the function $f\left(p_{b}, \alpha, p_{m}\right)$ is not dependent on $T$. The function $g\left(p_{m}, n\right)$ is non-linear and static and here represented by interpolation in a map. For more details on the model and especially identification and validation, see [3].

\subsection{Boost Leakage Model}

When a leak occurs, air will flow out of or into the airintake system depending on the air pressure compared to ambient pressure. In the engine used in this work, the boost pressure is during normal operation always higher than ambient pressure. This means that the air flow through a boost leak will always be in the direction out from the air tube. This air flow is modeled as an air flow through a restriction, like the model for flow past 


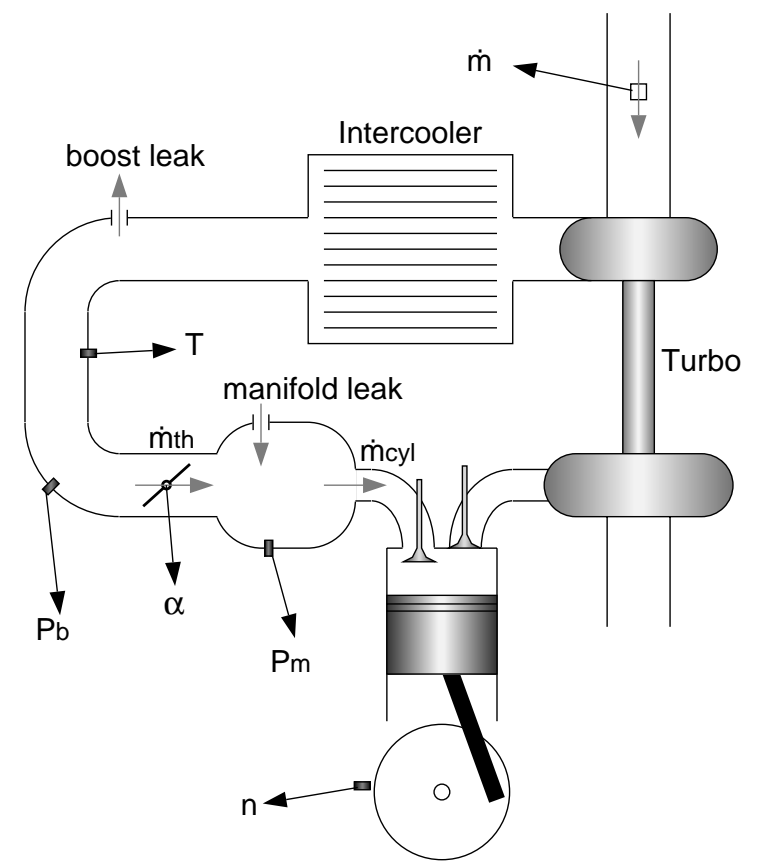

Figure 2 The turbo-charged engine. Air-mass flows that are discussed in the text are marked with gray arrows.

the throttle. The equation describing this air flow is

$$
\dot{m}_{\text {boostLeak }}=k_{b} h_{b}\left(p_{b}\right)=k_{b} \frac{p_{b}}{\sqrt{T}} \Psi\left(\frac{p_{a m b}}{p_{b}}\right)
$$

where it is assumed that the ambient pressure $p_{a m b}$ is constant. The parameter $k_{b}$ is proportional to the leakage area and therefore denoted equivalent area.

The model for the whole air-intake system with a boost leak present is obtained by replacing Equation (3a) with

$$
\dot{m}=\dot{m}_{t h}+\dot{m}_{\text {boostLeak }}
$$

\subsection{Manifold Leakage Model}

During most part of the operation of the engine, the manifold pressure is below ambient pressure. Therefore a manifold leak will mostly result in an air flow in the direction into the manifold. This flow is modeled in the same way as the model of flow through boost leaks, i.e.

$$
\dot{m}_{\text {manLeak }}=k_{m} h_{m}\left(p_{m}\right)=k_{m} \frac{p_{a m b}}{\sqrt{T_{a m b}}} \Psi\left(\frac{p_{m}}{p_{a m b}}\right)
$$

The model for the whole air-intake system with manifold leak present is obtained by replacing Equation (3a) with

$$
\dot{m}_{t h}+\dot{m}_{\text {manLeak }}=\dot{m}_{c y l}
$$

In the case the manifold pressure is higher than ambient pressure, which can occur because of the turbo-charger, the leak air-flow will be in the opposite direction. This means that the term $\dot{m}_{\text {manLeak }}$ in (6) will change sign and $p_{a m b}$ and $p_{m}$ in (5) are interchanged.

Both leakage models were validated in [3] and good agreement with real data was obtained.

\section{DIAGNOSIS SYSTEM}

This section presents the design of a diagnosis system in accordance with the principles discussed in Section 2. The objective is not to present a complete design but rather to give some examples that illustrates solutions for some typical cases. The different fault modes that will be considered are listed in Table 1. As seen, only fault modes consisting of single faults are considered. This corresponds to an assumption that only one fault can be present at the same time. The definitions of each fault mode, i.e. $\mathcal{M}_{\gamma}\left(\theta_{\gamma}\right)$, are given in Section 5.2, where the construction of the hypothesis tests are described.

\begin{tabular}{|l|l|}
\hline No Fault & $N F$ \\
Boost Leak & $B L$ \\
Manifold Leak & $M L$ \\
Boost Pressure Sensor Bias & $B B$ \\
Manifold Pressure Sensor Gain-Fault & $M G$ \\
Manifold Pressure Sensor Cut-Off & $M C$ \\
Throttle Sensor Linear Fault & $T L F$ \\
Air Mass-Flow Sensor Loose Contact & $A L C$ \\
Boost Pressure Sensor Arbitrary Fault & $B A F$ \\
\hline
\end{tabular}

Table 1 The fault modes considered.

\subsection{Hypothesis Tests}

To develop the actual hypothesis tests, we first need to decide the set of hypotheses to test. Because of the single fault assumption, it is natural to use one hypothesis test for each fault mode. Thus the set of hypothesis tests can be indexed by $\gamma$, i.e. $H T_{\gamma}$, and becomes

$$
\begin{aligned}
& H_{\gamma}^{0}: F_{p} \in M_{\gamma} \\
& H_{\gamma}^{1}: F_{p} \in M_{\gamma}^{C} \\
& \gamma \in\{N F, B L, M L, B B, M G, M C, T L F, A L C, B A F\}
\end{aligned}
$$

By using the definition of the sets $\Theta_{\gamma}$, these hypothesis tests can also be written

$$
\begin{aligned}
& H_{\gamma}^{0}: \theta \in \Theta_{\gamma} \\
& H_{\gamma}^{1}: \theta \in \Theta_{\gamma}^{C}
\end{aligned}
$$

It turns out that for most fault modes, the limit when the fault size goes to zero is equal to the fault mode "no fault". For example, a very small leakage is in practice the same as "no fault". This means that when fault mode 
$N F$, i.e. no fault, is present, most null hypothesis can not be rejected. The implication is that almost all sets $M_{\gamma}$ must include $N F$.

For each hypothesis test $\mathrm{HT}_{\gamma}$, we need to find a test quantity (often also called test statistic) and a rejection region. The sample data $\mathbf{x}$ for each hypothesis is

$$
\mathbf{x}=\left[\begin{array}{llll}
u\left(t_{0}\right) & u\left(t_{0}+1\right) & \ldots & u\left(t_{0}+N\right) \\
y\left(t_{0}\right) & y\left(t_{0}+1\right) & \ldots & y\left(t_{0}+N\right)
\end{array}\right]
$$

To simplify the notation, we have assumed that unit sample-time is used. The test quantity is a function $T_{\gamma}(\mathbf{x})$ from the sample data $\mathbf{x}$, to a scalar value which is to be thresholded by a threshold $J_{\gamma}$. Thus the rejection region is defined implicitly by the threshold $J_{\gamma}$ together with the test quantity $T_{\gamma}(\mathbf{x})$. The hypothesis test $\mathrm{HT}_{\gamma}$ is then defined as

$$
\begin{array}{ll}
T_{\gamma}(\mathbf{x})>J_{\gamma} & \text { reject } H_{\gamma}^{0}, \text { i.e. accept } H_{i}^{1} \\
T_{\gamma}(\mathbf{x})<J_{\gamma} & \text { do not reject } H_{\gamma}^{0}
\end{array}
$$

This means that we need to design a test quantity $T_{\gamma}(\mathbf{x})$ such that it is low if the data $\mathbf{x}$ match the hypothesis $H_{\gamma}^{0}$, i.e. a fault mode in $M_{\gamma}$ can explain the data. Also if the data come from a fault mode not in $M_{\gamma}, T_{\gamma}(\mathbf{x})$ should be high.

As test quantity, we will use the following function:

$$
T_{\gamma}(\mathbf{x})=\min _{\theta_{\gamma} \in \Xi_{\gamma}} V_{\gamma}\left(\theta_{\gamma}, \mathbf{x}\right)
$$

where $V_{\gamma}\left(\theta_{\gamma}, \mathbf{x}\right)$ is a norm measuring the validity of the model $\mathcal{M}_{\gamma}\left(\theta_{\gamma}\right)$ with respect to the data $\mathbf{x}$. To construct this norm, several principles are possible. One possibility is to use a sum of prediction errors and if there is statistical knowledge, also the likelihood function can be used. Since the modeling work, presented in Section 4, does not include statistical assumptions, it is in this work natural to use test quantities based on a sum of prediction errors.

In the special case when the fault mode $\gamma$ contains only one specific fault, i.e. the fault mode corresponds to one specific value of $\theta$, then the model is written $\mathcal{M}_{\gamma}$ and the test quantities becomes

$$
T_{\gamma}(\mathbf{x})=V_{\gamma}(\mathbf{x})
$$

\subsection{Description of the Hypothesis Tests}

In conclusion, the problem of designing hypothesis test $H T_{\gamma}$ consists of determining $V_{\gamma}\left(\theta_{\gamma}, \mathbf{x}\right), \Xi_{\gamma}$, and $J_{\gamma}$. Below we present the design of $V_{\gamma}\left(\theta_{\gamma}, \mathbf{x}\right)$ and $\Xi_{\gamma}$ for all hypothesis tests corresponding to the fault modes in Table 1 . The fault state vector considered is

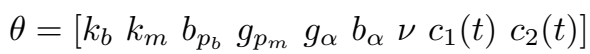

\section{No Fault $N F$}

The model $\mathcal{M}_{N F}$ corresponding to this fault mode is obtained by using the fault-free model (3) in combination with

$$
\begin{aligned}
\dot{m}_{s} & =\dot{m} \\
p_{b, s} & =p_{b} \\
p_{m, s} & =p_{m} \\
\alpha_{s} & =\alpha \\
n_{s} & =n
\end{aligned}
$$

where the index $s$ denotes that for example $\dot{m}_{s}$ is the sensor signal in contrast to $\dot{m}$ which is the physical quantity. The fault mode $N F$ corresponds to one specific value of $\theta$ which, in accordance with (11), means that $T_{N F}(\mathbf{x})=V_{N F}(\mathbf{x})$. The norm $V_{N F}(\mathbf{x})$ is defined as

$$
\begin{aligned}
V_{N F}(\mathbf{x})= & \frac{1}{N} \sum_{t=1}^{N}\left(\dot{m}_{s}-f\left(p_{b, s}, \alpha_{s}, p_{m, s}\right)\right)^{2}+ \\
& +\frac{1}{N} \sum_{t=1}^{N}\left(f\left(p_{b, s}, \alpha_{s}, p_{m, s}\right)-g\left(p_{m, s}, n_{s}\right)\right)^{2}
\end{aligned}
$$

Note that, to simplify notation, we have dropped the timeargument of signals. Using this norm implies that if the present fault mode is $N F$, the test quantity becomes small and for all other fault modes, the test quantity becomes large. In a hypothesis test defined by (7) and (9) this means that $M_{N F}=\{N F\}$.

\section{Boost Leak $B L$}

The model $\mathcal{M}_{B L}\left(k_{b}\right)$ corresponding to this fault mode is obtained by using the model described in Section 4.2 together with the identities (12). The scalar parameter $k_{b}$ defines the equivalent area of the leakage and is constrained by $\left.\left.k_{b} \in \Xi_{B L}=\right] 0,0.5\right]$. The norm $V_{B L}\left(k_{b}, \mathbf{x}\right)$ is

$$
\begin{aligned}
V_{B L}\left(k_{b}, \mathbf{x}\right)= & \frac{1}{N} \sum_{t=1}^{N}\left(\dot{m}_{s}-f\left(p_{b, s}, \alpha_{s}, p_{m, s}\right)-k_{b} h_{b}\left(p_{b, s}\right)\right)^{2}+ \\
& +\frac{1}{N} \sum_{t=1}^{N}\left(f\left(p_{b, s}, \alpha_{s}, p_{m, s}\right)-g\left(p_{m, s}, n_{s}\right)\right)^{2}
\end{aligned}
$$

Using this norm means that $M_{B L}=\{N F, B L\}$. The expression of $V_{B L}\left(k_{b}, \mathbf{x}\right)$ contains two terms even though only one of them is affected by the fault. The reason to include the second term is, as was said in the end of Section 5.1, that we want the test quantity to become large for other faults, not belonging to fault mode $B L$.

\section{Manifold Leak $M L$}

The model $\mathcal{M}_{M L}\left(k_{m}\right)$ is obtained in analogy with $\mathcal{M}_{B L}\left(k_{b}\right)$. The scalar parameter $k_{m}$ is constrained by 
$\left.\left.k_{m} \in \Xi_{M L}=\right] 0,0.5\right]$ and the norm $V_{M L}\left(k_{m}, \mathbf{x}\right)$ is

$$
\begin{gathered}
V_{M L}\left(k_{m}, \mathbf{x}\right)=\frac{1}{N} \sum_{t=1}^{N}\left(\dot{m}_{s}-f\left(p_{b, s}, \alpha_{s}, p_{m, s}\right)\right)^{2}+ \\
+\frac{1}{N} \sum_{t=1}^{N}\left(f\left(p_{b, s}, \alpha_{s}, p_{m, s}\right)-g\left(p_{m, s}, n_{s}\right)+k_{m} h_{m}\left(p_{m, s}\right)\right)^{2}
\end{gathered}
$$

Using this norm means that $M_{M L}=\{N F, M L\}$.

\section{Boost Pressure Sensor Bias $B B$}

The model $\mathcal{M}_{B B}\left(b_{p_{b}}\right)$ corresponding to this fault mode is obtained by using the fault free model (3) together with identities (12) but replacing (12b) with $p_{b, s}=p_{b}+b_{p_{b}}$. The scalar parameter $b_{p_{b}}$ is constrained by $b_{p_{b}} \in \Xi_{B B}=$ $[-30,0[\cup] 0,30]$ and the norm $V_{B B}\left(b_{p_{b}}, \mathbf{x}\right)$ is

$$
\begin{gathered}
V_{B B}\left(b_{p_{b}}, \mathbf{x}\right)=\frac{1}{N} \sum_{t=1}^{N}\left(\dot{m}_{s}-f\left(p_{b, s}-b_{p_{b}}, \alpha_{s}, p_{m, s}\right)\right)^{2}+ \\
\left.+\frac{1}{N} \sum_{t=1}^{N}\left(f\left(p_{b, s}-b_{p_{b}}, \alpha_{s}, p_{m, s}\right)-g\left(p_{m, s}, n_{s}\right)\right)\right)^{2}
\end{gathered}
$$

Using this norm means that $M_{B B}=\{N F, B B\}$.

\section{Manifold Pressure Sensor Gain-Fault $M G$}

The model $\mathcal{M}_{M G}\left(g_{p_{m}}\right)$ corresponding to this fault mode is obtained by using the fault free model (3) together with identities (12) but replacing (12c) with $p_{m, s}=g_{p_{m}} p_{m}$. The scalar parameter $g_{p_{m}}$ is constrained by $g_{p_{m}} \in \Xi_{M G}=$ $[0.5,1[\cup] 1,2]$ and the norm $V_{M G}\left(g_{p_{m}}, \mathbf{x}\right)$ is

$$
\begin{gathered}
V_{M G}\left(g_{p_{m}}, \mathbf{x}\right)=\frac{1}{N} \sum_{t=1}^{N}\left(\dot{m}_{s}-f\left(p_{b, s}, \alpha_{s}, p_{m, s} / g_{p_{m}}\right)\right)^{2}+ \\
\left.+\frac{1}{N} \sum_{t=1}^{N}\left(f\left(p_{b, s}, \alpha_{s}, p_{m, s} / g_{p_{m}}\right)-g\left(p_{m, s} / g_{p_{m}}, n_{s}\right)\right)\right)^{2}
\end{gathered}
$$

Using this norm means that $M_{M G}=\{N F, M G\}$.

\section{Manifold Pressure Sensor Cut-Off $M C$}

This fault mode represents a cut-off in the electrical connection to the manifold pressure sensor. The model $\mathcal{M}_{M C}(\nu)$ corresponding to this fault mode is obtained by using the fault free model (3) together with identities (12) but replacing $(12 \mathrm{c})$ with $p_{m, s}=\nu g_{p_{m}}$. The scalar parameter $\nu$ takes value 1 in the fault-free case and value 0 when there is a cut-off present. This means that $\Xi_{M C}=\{0\}$. This fault mode corresponds to exactly one value of $\theta$ which implies that $T_{M C}(\mathbf{x})=V_{M C}(\mathbf{x})$. The norm $V_{M C}(\mathbf{x})$ is defined as

$$
V_{M C}(\mathbf{x})=\frac{1}{N} \sum_{t=1}^{N} p_{b, s}^{2}
$$

Using this norm means that $M_{M C}=\{M C\}$. Note that, in spite of its simpleness, this test quantity is very large for all $\theta \notin \Theta_{M C}$. i.e. faults not belonging to $M C$. This is the reason why the fault mode $N F$ is not included in $M_{M C}$.

\section{Throttle Sensor Linear Fault $T L F$}

The model $\mathcal{M}_{T L F}\left(\left[\begin{array}{ll}g_{\alpha} & b_{\alpha}\end{array}\right]\right)$ corresponding to this fault mode is obtained by using the fault free model (3) together with identities (12) but replacing (12d) with $\alpha_{s}=$ $g_{\alpha} \alpha+b_{\alpha}$. The vector valued parameter $\left[\begin{array}{ll}g_{\alpha} & b_{\alpha}\end{array}\right]$ is constrained by $\left[g_{\alpha} b_{\alpha}\right] \in \Xi_{T L F}=\mathbb{R}^{2}-\{1,0\}$ and the norm $V_{T L F}\left(\left[g_{\alpha} b_{\alpha}\right], \mathbf{x}\right)$ is

$$
\begin{aligned}
& V_{T L F}\left(\left[g_{\alpha} b_{\alpha}\right], \mathbf{x}\right)= \\
& =\frac{1}{N} \sum_{t=1}^{N}\left(\dot{m}_{s}-f\left(p_{b, s},\left(\alpha_{s}-b_{\alpha}\right) / g_{\alpha}, p_{m, s}\right)\right)^{2}+ \\
& \left.+\frac{1}{N} \sum_{t=1}^{N}\left(f\left(p_{b, s},\left(\alpha_{s}-b_{\alpha}\right) / g_{\alpha}, p_{m, s}\right)-g\left(p_{m, s}, n_{s}\right)\right)\right)^{2}
\end{aligned}
$$

Using this norm means that $M_{T L F}=\{N F, T L F\}$.

\section{Air Mass-Flow Sensor Loose Contact $A L C$}

The model $\mathcal{M}_{A L C}\left(c_{1}(t)\right)$ corresponding to this fault mode is obtained by using the fault free model (3) together with identities (12) but replacing (12a) with $\dot{m}_{s}(t)=\dot{m}(t) c_{1}(t)$. The parameter $c_{1}(t)$ is a stochastic process taking values such that $c_{1}(t) \in\{0,1\}$. This means that the parameter space $\Xi_{A L C}$ becomes $\Xi_{M G}=\{0,1\}^{N}-\{1\}^{N}$ and the norm $V_{A L C}\left(c_{1}(t), \mathbf{x}\right)$ is

$$
\begin{array}{r}
V_{A L C}\left(c_{1}(t), \mathbf{x}\right)=\frac{1}{N} \sum_{t=1}^{N}\left(\dot{m}_{s}-c_{1} f\left(p_{b, s}, \alpha_{s}, p_{m, s}\right)\right)^{2}+ \\
\left.+\frac{1}{N} \sum_{t=1}^{N}\left(f\left(p_{b, s}, \alpha_{s}, p_{m, s}\right)-g\left(p_{m, s}, n_{s}\right)\right)\right)^{2}
\end{array}
$$

Using this norm means that $M_{A L C}=\{N F, A L C\}$

\section{Boost Pressure Sensor Arbitrary Fault (HT $\mathbf{H A F}_{\text {) }}$}

The model $\mathcal{M}_{M G}\left(c_{2}(t)\right)$ corresponding to this fault mode is obtained by using the fault free model (3) together with identities (12) but replacing (12b) with $p_{b, s}=p_{b}+c_{2}(t)$. The parameter $c_{2}(t)$ is a stochastic process taking arbitrary values. This means that the parameter space $\Xi_{B A F}$ 
becomes $\Xi_{B A F}=\mathbb{R}^{N}-\{0\}^{N}$ and the norm $V_{B A F}\left(c_{2}(t), \mathbf{x}\right)$ is

$$
\begin{gathered}
V_{B A F}\left(c_{2}(t), \mathbf{x}\right)=\frac{1}{N} \sum_{t=1}^{N}\left(\dot{m}_{s}-f\left(p_{b, s}-c_{2}, \alpha_{s}, p_{m, s}\right)\right)^{2}+ \\
\left.+\frac{1}{N} \sum_{t=1}^{N}\left(f\left(p_{b, s}-c_{2}, \alpha_{s}, p_{m, s}\right)-g\left(p_{m, s}, n_{s}\right)\right)\right)^{2}
\end{gathered}
$$

Using this norm means that $M_{B A F}=\{N F, B A F\}$.

\subsection{Practical Considerations}

The procedure to compute (10), i.e. to minimize $V_{\gamma}(\mathbf{x})$, has not been addressed so far. In many cases the minimization procedure required is quite straightforward. The technical details are not going to be discussed here, but the interested reader is referred to general optimization literature, e.g. [8], (see also the discussion in Section 7). However, for some of the hypothesis tests defined above, the computational load of doing the actual minimization in (10) can be quite heavy. One solution is to use a two-step procedure presented below.

First find a $\hat{\theta}_{\gamma}$ that minimizes another function $\bar{V}_{\gamma}\left(\theta_{\gamma}, \mathbf{x}\right)$, i.e.

$$
\hat{\theta}_{\gamma}=\arg \min _{\theta_{\gamma} \in \Xi_{\gamma}} \bar{V}_{\gamma}\left(\theta_{\gamma}, \mathbf{x}\right)
$$

Then use as test quantity $T_{\gamma}(\mathbf{x})=V_{\gamma}\left(\hat{\theta}_{\gamma}, \mathbf{x}\right)$.

If $\bar{V}_{\gamma}\left(\theta_{\gamma}, \mathbf{x}\right)$ is chosen such that the minimizing value $\hat{\theta}_{\gamma}$, under $H_{\gamma}^{0}$, is close to the value that minimizes $V_{\gamma}\left(\theta_{\gamma}, \mathbf{x}\right)$, then it is reasonable to assume that

$$
\min _{\theta_{\gamma} \in \Xi_{\gamma}} V_{\gamma}\left(\theta_{\gamma}, \mathbf{x}\right) \approx V_{\gamma}\left(\hat{\theta}_{\gamma}, \mathbf{x}\right)
$$

This means that if we use the test quantity $T_{\gamma}(\mathbf{x})=$ $V_{\gamma}\left(\hat{\theta}_{\gamma}, \mathbf{x}\right)$, we can expect approximately the same result compared to if (10) was used.

In the implementation of the the hypothesis tests defined above, this two-step procedure is used in the tests $\mathrm{HT}_{B L}, \mathrm{HT}_{M L}, \mathrm{HT}_{A L C}$, and $\mathrm{HT}_{B A F}$. In all these four cases, $\bar{V}_{\gamma}\left(\theta_{\gamma}, \mathbf{x}\right)$ is chosen as one of the two terms in $V_{\gamma}\left(\theta_{\gamma}, \mathbf{x}\right)$.

For $\mathrm{HT}_{B L}$ and $\mathrm{HT}_{M L}, \bar{V}_{\gamma}\left(\theta_{\gamma}, \mathbf{x}\right)$ is

$$
\bar{V}_{B L}\left(k_{b}, \mathbf{x}\right)=\frac{1}{N} \sum_{t=1}^{N}\left(\dot{m}_{s}-f\left(p_{b, s}, \alpha_{s}, p_{m, s}\right)-k_{b} h\left(p_{b, s}\right)\right)^{2}
$$

and

$$
\bar{V}_{M L}\left(k_{m}, \mathbf{x}\right)=\frac{1}{N} \sum_{t=1}^{N}\left(\dot{m}_{s}-f\left(p_{b, s}, \alpha_{s}, p_{m, s}\right)\right)^{2}
$$

respectively. For the test $\mathrm{HT}_{A L C}, \bar{V}_{A L C}\left(c_{1}(t), \mathbf{x}\right)$ is

$$
V_{A L C}\left(c_{1}(t), \mathbf{x}\right)=\frac{1}{N} \sum_{t=1}^{N}\left(\dot{m}_{s}-c_{1} f\left(p_{b, s}, \alpha_{s}, p_{m, s}\right)\right)^{2}
$$

This function can be conveniently minimized by choosing

$$
c_{1}(t)= \begin{cases}0 & \dot{m}_{s}(t)<\epsilon \\ 1 & \dot{m}_{s}(t) \geq \epsilon\end{cases}
$$

For the test $\mathrm{HT}_{B A F}, \bar{V}_{B A F}\left(c_{2}(t), \mathbf{x}\right)$ is

$$
\bar{V}_{B A F}\left(c_{2}(t), \mathbf{x}\right)=\frac{1}{N} \sum_{t=1}^{N}\left(\dot{m}_{s}-f\left(p_{b, s}-c_{2}, \alpha_{s}, p_{m, s}\right)\right)^{2}
$$

This function is conveniently minimized by choosing

$$
c_{2}(t)=f^{-1}\left(\dot{m}_{s}(t), \alpha_{s}(t), p_{m, s}(t)\right)-p_{b, s}
$$

where $f^{-1}\left(\dot{m}_{s}(t), \alpha_{s}(t), p_{m, s}(t)\right)$ is the "inverse" of $f\left(p_{b, s}, \alpha_{s}, p_{m, s}\right)$ and gives an estimate of $p_{b, s}$.

\subsection{Discussion}

As seen in Section 5.2 above, faults can be modeled in a number of different ways. For some fault modes, i.e. $B L$, $M L, B B, M G$, the fault is modeled as a change in a continuous scalar parameter. The fault modes $M C$ and $T L F$ are examples in which the fault is modeled as a change in a discrete and multidimensional parameter respectively. In contrast to this, a fault belonging to the fault mode $B A F$ is modeled as an additive arbitrary signal. Then we have $A L C$, in which the fault is a signal, or a parameter, that jumps between two distinct values. Although not exemplified here, the well studied case of abrupt changing parameters [9] can also be naturally handled.

All these examples clearly show the large variety of fault models that can be used in conjunction with structured hypothesis tests. In fact, while in many other papers, only continuous scalar parameter or only additive arbitrary signals are considered, it is shown here that almost any kind of fault models can be handled and also within the same framework and same diagnosis system.

Many other approaches are based on decoupling of subsets of faults modeled as additive signals. Decoupling means that we make a test quantity (or residual) insensitive to some faults. It is realized that the principle of decoupling has here been generalized to include decoupling of faults modeled in arbitrary ways. For example, the test quantity $T_{B L}(\mathbf{x})$ will not respond to a boost leakage fault which is modeled as a parameter change.

\section{EXPERIMENTAL VALIDATION}

The diagnosis system described in the previous section was implemented in Matlab and tested extensively with the experimental setup described in Section 3. The leakage faults were implemented in hardware, according to Section 3, and all other faults were emulated in software by applying appropriate changes to the sensor signals. For 
each fault mode, a number of different fault sizes were tested.

Good functionality was obtained for all kinds of faults but because of space limitation, we have selected only four cases which are shown in Table 2 to 5 . These four cases are not selected because they are representative but rather because they illustrates some interesting features of the diagnosis system. In all these cases, the data length was $N=1000$ which corresponds to $100 \mathrm{~s}$. No special effort was made to find optimal threshold values $J_{\gamma}$; they were all chosen to be $J_{\gamma}=0.4$.

\subsection{Fault Mode $N F$}

In Table 2, the present fault mode of the process was NF. Each row show the result of one individual hypothesis test $H T_{\gamma}$. The value of the test quantity $T_{\gamma}(\mathbf{x})$ for each hypothesis $H T_{\gamma}$ is shown in the second column. The threshold $J_{\gamma}$ is shown in the third column (in this example, all were chosen to the same value). If $T_{\gamma}(\mathbf{x})>J_{\gamma}$, i.e. $H_{\gamma}^{0}$ is rejected, then the fourth column shows the the set $M_{\gamma}^{C}$.

For the case shown in the table, only the null hypothesis $H_{M C}^{0}$ is rejected. This result is the one expected because the set $M_{M C}$ do not contain the fault mode $N F$ while all other sets $M_{\gamma}$ do contain $N F$. Applying the intersection of the decision logic, i.e. (2), implies that the diagnosis statement contains 8 possible fault modes that can explain the behavior of the process. One of the fault modes is $N F$ which means that we should not generate an alarm.

\begin{tabular}{llll}
$\gamma$ & $T_{\gamma}(\mathbf{x})$ & $J_{\gamma}$ & $M_{\gamma}^{C}$ \\
\hline NF & 0.2074 & 0.4 & \\
BL & 0.2063 & 0.4 & \\
ML & 0.2075 & 0.4 & \\
BB & 0.2043 & 0.4 & \\
MG & 0.2027 & 0.4 & \\
MC & 3608 & 0.4 & ALC BAF BB BL MG ML NF TLF \\
TLF & 0.2061 & 0.4 & \\
ALC & 0.2074 & 0.4 & \\
BAF & 0.1491 & 0.4 & \\
\hline Diagn. Statement: & ALC BAF BB BL MG ML NF TLF \\
no alarm
\end{tabular}

Table 2 The hypothesis tests and the diagnosis statement for fault mode $N F$.

\subsection{Fault Mode $T L F$}

In Table 3 the present fault mode of the process was $T L F$. Now all individual null hypothesis are rejected except for $H_{T L F}^{0}$. The diagnosis statement is the single fault mode $T L F$. Because the diagnosis statement does not contain $N F$, an alarm is generated.

\begin{tabular}{llll}
$\gamma$ & $T_{\gamma}(\mathbf{x})$ & $J_{\gamma}$ & $M_{\gamma}^{C}$ \\
\hline NF & 250.8 & 0.4 & ALC BAF BB BL MC MG ML TLF \\
BL & 170.7 & 0.4 & ALC BAF BB MC MG ML TLF \\
ML & 230.2 & 0.4 & ALC BAF BB BL MC MG TLF \\
BB & 247 & 0.4 & ALC BAF BL MC MG ML TLF \\
MG & 175.6 & 0.4 & ALC BAF BB BL MC ML TLF \\
MC & 3608 & 0.4 & ALC BAF BB BL MG ML NF TLF \\
TLF & 0.2025 & 0.4 & \\
ALC & 250.8 & 0.4 & BAF BB BL MC MG ML TLF \\
BAF & 273.7 & 0.4 & ALC BB BL MC MG ML TLF \\
\hline
\end{tabular}

Diagnosis Statement: TLF

ALARM

Table 3 The hypothesis tests and the diagnosis statement for fault mode $T L F$.

\subsection{Fault Mode $M L$}

In Table 4 the fault mode of the process was $M L$. The actual fault was fairly small, which is reflected in the result that it could not be uniquely isolated. The diagnosis statement contains the fault modes $M G, M L$, and $T L F$. This should be interpreted as that in addition to the present fault mode $M L$, the fault modes $M G$ and $T L F$ can also explain the behavior of the process. Because the fault statement does not contain $N F$, an alarm is generated.

\begin{tabular}{llll}
$\gamma$ & $T_{\gamma}(\mathbf{x})$ & $J_{\gamma}$ & $M_{\gamma}^{C}$ \\
\hline NF & 0.4921 & 0.4 & ALC BAF BB BL MC MG ML TLF \\
BL & 0.4985 & 0.4 & ALC BAF BB MC MG ML TLF \\
ML & 0.1881 & 0.4 & \\
BB & 0.423 & 0.4 & ALC BAF BL MC MG ML TLF \\
MG & 0.328 & 0.4 & \\
MC & 3742 & 0.4 & ALC BAF BB BL MG ML NF TLF \\
TLF & 0.3623 & 0.4 & \\
ALC & 0.4921 & 0.4 & BAF BB BL MC MG ML TLF \\
BAF & 0.4642 & 0.4 & ALC BB BL MC MG ML TLF \\
\hline
\end{tabular}

Diagnosis Statement: MG ML TLF

ALARM

Table 4 The hypothesis tests and the diagnosis statement for fault mode $M L$.

\subsection{Fault Mode $B B$}

In Table 5 the present fault mode of the process was $B B$. The actual fault was not very small but in spite of this, it is obvious from the diagnosis statement that the present fault mode $B B$ can not be uniquely isolated. The reason is that the fault mode $B A F$, which represent an arbitrary boost-pressure sensor fault, is so general that it can also explain data generated from the process when fault mode 
$B B$ is present. In this sense, the fault mode $B B$ is a subclass of the fault mode $B A F$. The space of $B B$ is very small compared to the space of $B A F$. Therefore, when both $B B$ and $B A F$ can explain the data, it is much more likely that the data has been generated by a process with fault mode $B B$. It is possible to extend the diagnosis system with this kind of reasoning, and in that case the fault statement would become the single fault mode $B B$.

\begin{tabular}{llll}
$\gamma$ & $T_{\gamma}(\mathbf{x})$ & $J_{\gamma}$ & $M_{\gamma}^{C}$ \\
\hline NF & 1.958 & 0.4 & ALC BAF BB BL MC MG ML TLF \\
BL & 1.96 & 0.4 & ALC BAF BB MC MG ML TLF \\
ML & 1.96 & 0.4 & ALC BAF BB BL MC MG TLF \\
BB & 0.2043 & 0.4 & \\
MG & 0.6725 & 0.4 & ALC BAF BB BL MC ML TLF \\
MC & 3608 & 0.4 & ALC BAF BB BL MG ML NF TLF \\
TLF & 0.419 & 0.4 & ALC BAF BB BL MC MG ML \\
ALC & 1.958 & 0.4 & BAF BB BL MC MG ML TLF \\
BAF & 0.1491 & 0.4 & \\
\hline
\end{tabular}

Diagnosis Statement: BAF BB

ALARM

Table 5 The hypothesis tests and the diagnosis statement for fault mode $B B$.

\section{ON-LINE IMPLEMENTATION}

For implementation in on-board diagnosis systems, on-line performance is crucial. The presentation of the diagnosis system so far has been made by assuming that, given data $\mathbf{x}$, the objective is to generate a diagnosis statement based on this data. This is sometimes referred to as an off-line approach but can equally well be implemented in an online fashion.

One on-line solution is to use a sliding time window such that the data $\mathbf{x}$ is defined as

$$
\mathbf{x}(t)=\left[\begin{array}{llll}
u(t-N) & u(t-N+1) & \ldots & u(t) \\
y(t-N) & y(t-N+1) & \ldots & y(t)
\end{array}\right]
$$

and thereby becomes a function of time $t$. Here $N$ is the length of the time window. It is possible to have a sliding time window and let to consecutive data sets be overlapping. Another choice is to let consecutive data sets be non-overlapping.

Although not discussed here, it is also possible to use a recursive approach. For example, the recursive procedure presented in [3] and based on the RLS (Recursive Least Square) algorithm [10], can be used to minimize $V_{B L}\left(k_{b}, \mathbf{x}\right)$ and $V_{M L}\left(k_{m}, \mathbf{x}\right)$ recursively. This problem is particularly simple because $k_{b}$ and $k_{m}$ enters linearly in the equations. For parameters entering non-linearly, other standard recursive techniques for parameter estimation can often be used, see [10].
In cases when the fault modes represents arbitrary faults, e.g. $B A F$, techniques based on observers can be used, e.g. see [11][12]. Especially for linear systems, these techniques have been extensively studied, e.g. see [13].

\subsection{On-Line Experiment}

To illustrate the performance in an on-line implementation, an experiment was setup. The fault mode of the process was $M G$ and the size of the fault was $g_{p_{m}}=1.2$. The whole data set (from the FTP75 test-cycle) spans over a time of 21 minutes. A non-overlapping window of length $N=100$ was used which corresponds to a time-length of $10 \mathrm{~s}$. This means that the original data set was divided in totally 125 smaller data sets.

It is common that the absolute accuracy of a model is dependent on how the system is excited and/or the input size. In these situations, an adaptive threshold can substantially improve the performance of the diagnosis system [14][15]. This is the case here and an adaptive threshold $J_{a d p}(t)$ was chosen as

$$
J_{a d p}(t)=\min _{\gamma} T_{\gamma}(\mathbf{x}(t))+0.05
$$

The first term serves as a measure of the overall accuracy of the model at time $t$ and the second term is a tuning parameter. This adaptive threshold was used in all hypothesis tests.

\begin{tabular}{|l|l|}
\hline NF & 0 \\
BL & 0 \\
ML & 57 \\
BB & 2 \\
MG & 120 \\
MC & 0 \\
TLF & 1 \\
ALC & 0 \\
BAF & 0 \\
unknown fault & 1 \\
\hline
\end{tabular}

Table 6 The number of occurrences of different fault modes in the diagnosis statement during the online experiment.

For all 125 data sets, the diagnosis system managed to detect a fault. The number of times each fault mode was contained in the diagnosis statement is shown in Table 6 . It is seen that except for that $M L$ was in the diagnosis statement 57 number of times, the performance was very good.

To understand why $M L$ occurs so many times in the diagnosis statement, Figure 3 has been included. The test quantities $T_{M L}(\mathbf{x}(t))$ and $T_{M G}(\mathbf{x}(t))$ are plotted together with the adaptive threshold $J_{a d p}$. Only data from time window \#50 to \#125 is shown. Ideally the test quantities 


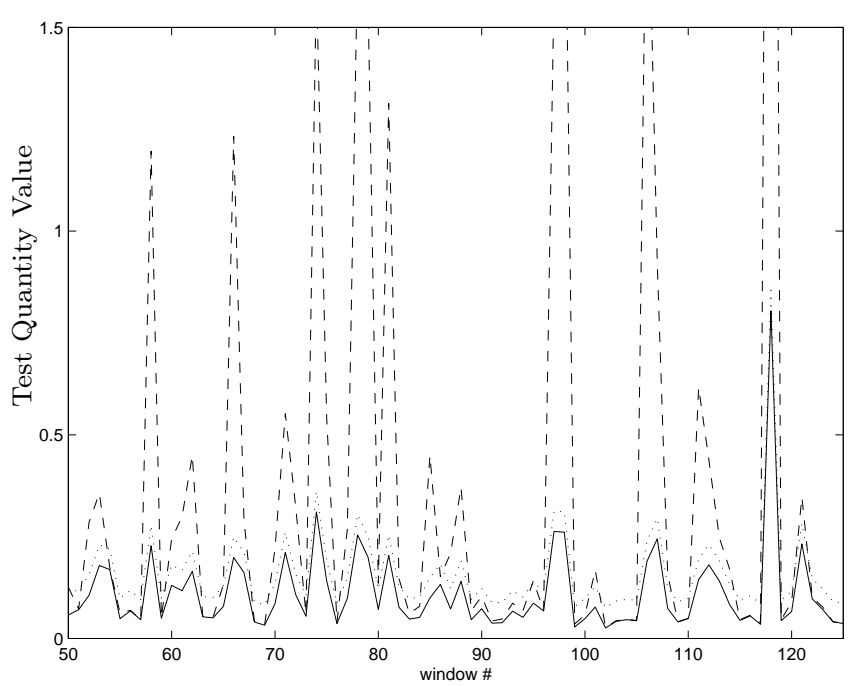

Figure 3 The test quantities $T_{M L}(\mathbf{x}(t))$ (dashed) and $T_{M G}(\mathbf{x}(t))$ (solid) together with the adaptive threshold $J_{\text {adp }}(t)$ (dotted).

$T_{M G}(\mathbf{x}(t))$ should be below the threshold and $T_{M L}(\mathbf{x}(t))$ should be above the threshold. This is the case most of the time but in some cases, both test quantities are below the threshold. These are the cases in which $M L$ is contained in the diagnosis statement.

From the figure it is obvious that for some states of the process, the test quantity $T_{M L}(\mathbf{x}(t))$ gets approximately the same value $T_{M G}(\mathbf{x}(t))$. This is probably due to a property of the air-intake system and not the diagnosis system. We can in that case not expect that $M L$ is, at all times, excluded from the diagnosis statement, no matter how the diagnosis system is designed.

\section{CONCLUSIONS}

Model based solutions to diagnosis problems in SI-engines have been discussed in many papers. However most methods are useful only for a specific class of faults. Here a more general method is presented. The method is based on hypothesis testing and has a potential to fully utilize the model of the system in a systematic way. With this method, it is possible to diagnose a large variety of different types of faults.

The method is applied to the diagnosis of sensor faults and leakage in the air-intake system of an SI-engine. Two previous methods, presented in [2] and [3], solved these two diagnosis problems by using two different methods. However, each of these methods is dedicated to a certain type of faults and the two methods can not easily be combined into one single diagnosis system capable of diagnosing both leaks and sensor faults.

In contrast, the new method, utilizing a structure of hypothesis tests, is capable of handling both leakage and different types of sensor faults. The features of the method are demonstrated by using experiments on a real SI-engine. The experiments show that the method is capable to diagnose both leakage and different types of sensor faults. This includes both detection and isolation. It is for example possible to distinguish between a manifold leak and a manifold pressure sensor fault.

\section{ACKNOWLEDGMENTS}

This research is supported by NUTEK (Swedish National Board for Industrial and Technical Development) and SAAB Automobile AB.

\section{REFERENCES}

[1] California's OBD-II regulation (section 1968.1, title 13, california code of regulations), resolution 93-40, july 9 . pages $220.7-220.12(\mathrm{~h}), 1993$.

[2] M. Nyberg and L. Nielsen. Model based diagnosis for the air intake system of the SI-engine. SAE Paper, (970209), 1997.

[3] M. Nyberg and A. Perkovic. Model based diagnosis of leaks in the air-intake system of an SI-engine. $S A E$ Paper, (980514), 1998.

[4] J. Gertler. Analytical redundancy methods in fault detection and isolation; survey and synthesis. IFAC Fault Detection, Supervision and Safety for Technical Processes, pages 9-21, Baden-Baden, Germany, 1991.

[5] E. Hendricks. Mean value modelling of spark ignition engines. SAE-Technical Paper Series, (900616), 1990.

[6] John B. Heywood. Internal Combustion Engine Fundamentals. McGraw-Hill series in mechanical engineering. McGraw-Hill, 1992.

[7] Charles Fayette Taylor. The Internal Combustion Engine in Theory and Practice. The M.I.T. Press, second edition, 1994.

[8] D. Luenberger. Linear and Nonlinear Programming. Addison Wesley, 1989.

[9] M. Basseville and I.V. Nikiforov. Detection of Abrupt Changes. PTR Prentice-Hall, Inc, 1993.

[10] Lennart Ljung. System Identification: Theory for the User. Prentice Hall, 1987.

[11] R.J. Patton. Robust model-based fault diagnosis:the state of the art. IFAC Fault Detection, Supervision and Safety for Technical Processes, pages 1-24, Espoo, Finland, 1994. 
[12] P.M. Frank. Advances in observer-based fault diagnosis. Proc. TOOLDIAG'93, pages 817-836, Toulouse, France, 1993. CERT.

[13] M. Nyberg and E. Frisk. A minimal polynomial basis solution to residual generation for fault diagnosis in linear systems. IFAC, 1999.

[14] R. Clark. State Estimation Schemes for Instrument Fault Detection, chapter 1. In (Patton et al., 1989). 1989.

[15] X. Ding and P.M. Frank. Frequency domain approach and threshold selector for robust model-based fault detection and isolation. IFAC Fault Detection, Supervision and Safety for Technical Processes, pages 271-276, Baden-Baden, Germany, 1991. 